\title{
A randomized trial of audio computer and in-person interview to assess HIV risk among drug and alcohol users in Rio De Janeiro, Brazil
}

\author{
Anna Azevedo Simoes, (M.D., Ph.D.) ${ }^{\mathrm{a}, *}$, Francisco Inacio Bastos, (M.D., Ph.D.) ${ }^{\mathrm{b}}$, \\ Ronaldo Ismerio Moreira, (M.Sc.) ${ }^{\mathrm{b}}$, Kevin G. Lynch, (Ph.D.) $)^{\mathrm{c}}$, David S. Metzger, (Ph.D.) ${ }^{\mathrm{c}}$ \\ ${ }^{a}$ Health Department of the State Government of Rio de Janeiro, Rio de Janeiro, Brazil \\ ${ }^{\mathrm{b}}$ Oswaldo Cruz Foundation, Rio de Janeiro, Brazil \\ ${ }^{\mathrm{c}}$ University of Pennsylvania, Philadelphia, PA, USA
}

Received 12 May 2005; received in revised form 10 December 2005; accepted 18 December 2005

\begin{abstract}
This study compares drug patterns and prevalence of risk behaviors in a randomized trial using two methods of administration, Audio Computer-Assisted Self-Interview (ACASI) and Interviewer-Administered Questionnaire (IAQ), among drug users seeking treatment in a drug treatment center. We randomized 735 participants: 367 to ACASI and 368 to IAQ. No significant difference in sociodemographic variables were found between subjects in the two arms of the study. Those interviewed by ACASI were more likely to report use on 7 of 10 substances assessed. Rates of reporting of sexual risk behaviors (male-to-male and commercial sex) were higher among participants in the ACASI arm. ACASI seems to be a key resource in improving the reporting of sensitive data in Brazil, as it has been in prior international studies. (C) 2006 Elsevier Inc. All rights reserved.
\end{abstract}

Keywords: ACASI; Substance abuse; Risk behavior; HIV/STI; Interview

\section{Introduction}

The assessment of behaviors associated with the transmission of HIV and other blood-borne and sexually transmitted infections is crucial in the design and evaluation of effective prevention interventions. Given the often illicit and personal nature of these transmission-related behaviors among drug users, the utility of such assessments depends on the ability to accurately measure what are often considered socially undesirable behaviors. A growing body of research suggests that accuracy in reporting such behaviors can be significantly enhanced by the use of computer-based interviewing commonly referred to as Audio Computer-Assisted Self-Interview (ACASI) (Davis

* Corresponding author. State Center of Treatment and Rehabilitation of Addicts, Rua D. Mariana 151, Botafogo, Rio de Janeiro, RJ 22280-020, Brazil. Tel.: +55 212299 5920; fax: +55 2122995922 .

E-mail address: annago@uol.com.br (A.A. Simoes).
\& Morse, 1991; Kissinger et al., 1999; Lucas, Mullin, Luna, \& McInroy, 1977; Mills et al., 1996; Millstein, 1987; Navaline et al., 1994; Newman et al., 2002). Collectively, the findings of these research studies suggest that respondents are more likely to report socially undesirable behaviors to a computer than to an interviewer. Furthermore, the use of ACASI strategy minimizes interviewer-introduced biases and eliminates error in the delivery of questions.

The utility of ACASI approach has been demonstrated in a variety of research settings and in a wide range of populations. As a research tool, the utility of the ACASI approach has been demonstrated among adolescents (Ellen et al., 2002; Jennings, Lucenko, Malow, \& Devieux, 2002; Mensch, Clark, \& Anh, 2003; Millstein \& Irwin, 1983; Turner et al., 1998), blood donors (Locke et al., 1992), needle exchange participants (Des Jarlais et al., 1999; Metzger et al., 2000; Riley et al., 2001), gay men (Gribble et al., 2000; Metzger et al., 2000), and injection drug users entering treatment (Perlis, Des Jarlais, Friedman, Arasteh, \& Turner, 
2004). Several features of the ACASI mode of questionnaire administration appear to contribute to its success in eliciting higher rates of reporting of socially undesirable behaviors. The respondent, independent of others, can view questions on a computer screen while listening (through headphones) to such questions as read by a recorded human voice. Thus, the pace of the interview is determined by the respondent; all respondents hear the questions read in the same way, and responses are simply and privately recorded through the use of a keyboard, a mouse, or a touch screen. ACASI can also be programmed to require a response to a question before proceeding forward, thus avoiding problems with missing data. Skip patterns are preprogrammed, avoiding confusion and error in the sequence of question administration. Because no aspect of the procedure requires the ability to read or write, ACASI can be used with individuals with limited literacy.

Although there is substantial literature on the utility of ACASI, few studies have tested the ability of ACASI to enhance the reporting of socially undesirable behaviors outside the United States. A recent literature review (Simoes \& Bastos, 2004) found only three studies of ACASI conducted in other countries (Sattah et al., 2002; Van de Wijgert, Padian, Shiboski, \& Turner, 2000; Van Griensven et al., 2001). Of these, only one was found to have tested the ability of ACASI to enhance the reporting of sensitive behaviors (Van de Wijgert et al., 2000). Because it is plausible that the improved reporting found while using ACASI is context-specific or culturally specific, there is a need for research to evaluate the generalizability of these findings to other cultural settings.

This study is the first to use the ACASI method in Brazil. In this article, we report on the results of a randomized trial comparing ACASI to Interviewer-Administered Questionnaires (IAQs). The questionnaire assessed recent drug use and risk behaviors among drug users entering a specialized public facility for the treatment of drug use problems in Rio de Janeiro, Brazil. This outpatient facility, the Rio de Janeiro State Center for Treatment and Rehabilitation of Addicts (CENTRA-RIO), was founded in 1998. It provides treatment for a broad range of substance abuse problems and currently admits approximately 1,000 new patients per year. We hypothesized that the participants assigned to the ACASI method would report more drug use and sexual risk behaviors.

\section{Materials and methods}

\subsection{Participants}

The participants were recruited from individuals seeking treatment between September 2002 and October 2003. To be eligible for this study, individuals had to be at least 18 years of age (the Brazilian legal age for providing informed consent) and had to report the use of at least one drug during the month prior to the interview. Participants were excluded if they had any psychiatric conditions or cognitive impairments identified at the time of assessment (including those caused by recent drug use or withdrawal) that could alter their ability to understand the research or to participate in it. Prior to data collection, all participants received explanations regarding the purpose of the research and their roles as research subjects.

After eligibility had been checked and informed consent had been signed, subjects were randomly assigned (1:1 on an alternating sequential basis) to one of the two arms: ACASI or IAQ administration. The study staff were trained on both the administration of the questionnaire and the procedure for conducting the interview using the ACASI approach.

\subsection{Assessments}

The questionnaire used in this study was derived from risk assessment battery (RAB). The RAB is a brief structured interview used for assessing recent $(6$ months prior) risk behaviors among drug users and assesses four domains: (1) use of alcohol and others drugs; (2) drugrelated risks (use of needle, syringes, and other injection paraphernalia); (3) sexual risks; and (4) concerns about HIV (e.g., testing and infecting others). The English version of the RAB was translated into Portuguese and tested in a pilot study using an interviewer-administered format (Simoes et al., 2002). The resulting Portuguese version of the questionnaire contains 42 questions with categorical response options (e.g., not at all, a few times, a few times each week, or everyday) and takes approximately 15 minutes to complete. It was used in both modes of administration (computer-assisted and interviewer-administered). The paper version was printed according to the specifications of TELEform 6.1 (Cardiff, Vista, CA). TELEform 6.1 uses a specific format for question layout so that responses can be easily recorded by the interviewer and accurately read by scanning equipment.

The programming of the ACASI Portuguese version utilized software specifically designed for ACASI development. The system was designed so that participants indicated their responses to questions using a mouse. The ACASI version presents each question on a separated screen. ACASI was programmed so that the participant could not skip a question without choosing one of the response options. Each screen also provided an option "button" that allowed participants to repeat the question and response choices as many times as needed. Sound files were recorded using a female human voice with a pleasant professional tone and without any distinct accent. This decision reflects our aim to maximize clarity and our concern with some respondents' potential to be reactive to distinct accents.

Three staff members were trained to conduct the interview using the same questionnaire as programmed into ACASI. Interviewers were instructed to read the questions exactly as written, to avoid comments related to answers, or 
Table 1

Sociodemographic characteristics of the sample, by method of interview (CENTRA-RIO, Rio de Janeiro, Brazil, 2002-2003)

\begin{tabular}{|c|c|c|c|c|}
\hline Characteristics & $\begin{array}{l}\text { ACASI } \\
(n=367) \\
{[n(\%)]}\end{array}$ & $\begin{array}{l}\text { Face-to-face } \\
(n=368) \\
{[n(\%)]}\end{array}$ & $\begin{array}{l}\text { Total } \\
(N=735) \\
{[n]}\end{array}$ & $\chi^{2}$ \\
\hline \multicolumn{5}{|l|}{ Sex } \\
\hline Male & $310(84.2)$ & 315 (85.6) & 625 & \\
\hline Female & $58(15.8)$ & 53 (14.4) & 111 & 0.26 \\
\hline \multicolumn{5}{|l|}{ Age (years) } \\
\hline $18-25$ & 69 (18.8) & $70(19.0)$ & 139 & \\
\hline $26-35$ & $118(32.1)$ & $115(31.3)$ & 233 & \\
\hline $36-45$ & $120(32.6)$ & $105(28.5)$ & 225 & \\
\hline $46+$ & $61(16.6)$ & $78(21.1)$ & 139 & \\
\hline$M(S D)$ & $35.8(10.7)$ & $36.4(10.9)$ & $36.1(10.8)$ & 3.45 \\
\hline \multicolumn{5}{|l|}{ Race } \\
\hline White & $187(51.1)$ & $170(48.7)$ & 357 & \\
\hline Non-White & $179(48.9)$ & $196(51.2)$ & 375 & 2.58 \\
\hline \multicolumn{5}{|l|}{ Education } \\
\hline$<$ High school & $243(68.1)$ & $253(70.5)$ & 496 & \\
\hline$\geq$ High school & $114(31.9)$ & $106(29.5)$ & 220 & 0.48 \\
\hline \multicolumn{5}{|l|}{ Marital status } \\
\hline Single & $248(67.9)$ & $229(62.2)$ & 477 & 2.64 \\
\hline $\begin{array}{l}\text { Stable } \\
\text { partner/spouse }\end{array}$ & $117(32.1)$ & $139(37.8)$ & 256 & \\
\hline
\end{tabular}

to avoid discussions of reports of risk until after completion of the interview. All interviews were completed in a private room, with only the interviewer and the respondent present. If the respondent indicated uncertainty with any response, the interviewers were instructed to encourage the respondent to choose the response that most closely matched their perception of their behavior.

After randomization to the ACASI arm, the participant was seated in front of the computer, and the staff provided a brief training on how to use the system. Four practice questions with neutral content provided the staff the opportunity to observe and instruct the participant on answering questions with structures similar to those found in the questionnaire. When the participant felt comfortable in managing the computer, the staff member left the room but remained available for further questions on the operation of the computer.

\subsection{Statistical analyses}

The sample size was calculated to identify both statistical and clinically meaningful differences between responses to the computer (ACASI) and those reported to the interviewer. Statistical significance was assessed using both unadjusted odds ratios (ORs) and logistic regression analyses, with adjustment for age, gender, race, education, marital status, and income. A clinically meaningful difference was defined for this study as a $10 \%$ or greater rate of reporting on ACASI relative to the rate of reporting on the IAQ approach. For the assessment of clinical significance, we selected several key variables that are considered important indicators of risk - cocaine use, inconsistent condom use, men-having-sex-with-men (MSM) behavior, and exchange of sex for drugs or money. In determining power, we assumed that the rates of reporting of these behaviors to the IAQ approach would fall below $30 \%$ or above $80 \%$. Because our hypothesis was directional-that respondents would report higher rates of drug use and sexual risk behaviors to ACASI relative to IAQ - we calculated that a sample size of 280 subjects per group would provide us sufficient power to detect differences of $10 \%$ or greater between the groups with $80 \%$ power $(1-\beta)$ and $\alpha=0.05$.

Data entry was processed in two ways. (1) The interviewer-administered version of RAB, the sociodemographic interview, and the laboratory forms processed using TELEform 6.1 were faxed (once a week) by the staff to our central database, which used a fax/modem board to incorporate the data into the database system. (2) The ACASI version stored the data of each interview into the hard drive of a laptop. At the end of each day, the staff copied the data into a diskette. Once a week, ACASI data were sent directly to our database system by Internet.

Table 2

Drug use reported 30 days prior to the interview, by method of interview (CENTRA-RIO, Rio de Janeiro, Brazil, 2002-2003)

\begin{tabular}{|c|c|c|c|c|c|}
\hline Drugs & $\begin{array}{l}\text { ACASI } \% \text { affirmative } \\
\text { answer }[n(\%)]\end{array}$ & $\begin{array}{l}\text { Face-to-face } \% \text { affirmative } \\
\text { answer }[n(\%)]\end{array}$ & OR & Adjusted OR ${ }^{\mathrm{a}}$ & $\begin{array}{l}95 \% \text { Confidence } \\
\text { interval }\end{array}$ \\
\hline Alcohol & $326(88.6)$ & $322(87.5)$ & 1.10 & 1.18 & $0.74-2.01$ \\
\hline Marijuana & $153(41.6)$ & $132(35.9)$ & 1.27 & 1.35 & $0.97-1.88$ \\
\hline Cocaine, snorted & $223(60.6)$ & $185(50.3)$ & 1.52 & $1.63^{*}$ & $1.18-2.25$ \\
\hline Cocaine, intravenous & $32(8.7)$ & $4(1.1)$ & 8.66 & $8.60^{*}$ & $2.99-24.76$ \\
\hline Heroin, intravenous & 7 (1.9) & $0(0)$ & - & - & - \\
\hline Crack & $26(7.1)$ & $13(3.5)$ & 2.07 & $2.11 *$ & $1.04-4.24$ \\
\hline Amphetamine, oral & $15(4.1)$ & $1(0.3)$ & 15.57 & $15.73 *$ & $2.05-120.34$ \\
\hline Benzodiazepines & $92(25.0)$ & $53(14.4)$ & 1.98 & $1.92 *$ & $1.31-2.82$ \\
\hline Opioids, oral & $70(19.0)$ & $39(10.6)$ & 1.98 & $1.93 *$ & $1.25-2.98$ \\
\hline Cough syrup, intravenous & $37(10.1)$ & $1(0.3)$ & 40.85 & $40.85^{*}$ & $5.37-292.70$ \\
\hline Hallucinogens & $22(6.0)$ & $6(1.6)$ & 3.81 & $3.81 *$ & $1.47-9.31$ \\
\hline
\end{tabular}

${ }^{a}$ Results from a logistic regression analysis controlling for gender, age, education, race, and marital status.

$* p<.05$. 
Table 3

Sexual behavior reported 6 months prior to the interview, by method of interview (CENTRA-RIO, Rio de Janeiro, Brazil, 2002-2003)

\begin{tabular}{|c|c|c|c|c|c|}
\hline Sexual behavior & $\begin{array}{l}\text { ACASI } \% \text { affirmative } \\
\text { answer }[n(\%)]\end{array}$ & $\begin{array}{l}\text { Face-to-face } \% \text { affirmative } \\
\text { answer }[n(\%)]\end{array}$ & OR & Adjusted $\mathrm{OR}^{\mathrm{a}}$ & $\begin{array}{l}95 \% \text { Confidence } \\
\text { interval }\end{array}$ \\
\hline Sexually active & $332(90.2)$ & $338(91.8)$ & 0.81 & 0.79 & $0.47-1.35$ \\
\hline Number of partners $\geq 2$ & $172(51.8)$ & $178(52.7)$ & 0.96 & 0.91 & $0.65-1.27$ \\
\hline MSM behavior & $39(12.6)$ & $18(5.7)$ & $2.37 *$ & $2.52 *$ & $1.38-4.61$ \\
\hline Sex for drugs & $67(18.2)$ & $40(10.9)$ & $1.82 *$ & $1.88^{*}$ & $1.20-2.94$ \\
\hline Drugs for sex & $75(20.4)$ & $60(16.3)$ & 1.31 & 1.30 & $0.87-1.93$ \\
\hline Money for sex & $97(26.4)$ & $74(20.1)$ & $1.42 *$ & $1.37 *$ & $0.95-1.98$ \\
\hline Sex with $\mathrm{HIV}^{+}$partner & $10(2.7)$ & $9(2.4)$ & 1.11 & 1.32 & $0.51-3.42$ \\
\hline Inconsistent condom use & $279(83.0)$ & $260(78.5)$ & 1.33 & 1.34 & $0.90-2.01$ \\
\hline Concern about being exposed to HIV & $231(61.0)$ & $213(58.4)$ & $1.37^{*}$ & 1.32 & $0.96-1.82$ \\
\hline Had been tested for HIV & $149(42.9)$ & $167(45.9)$ & 0.88 & 0.85 & $0.6-1.2$ \\
\hline
\end{tabular}

${ }^{\text {a }}$ Results from a logistic regression analysis controlling for gender, age, education, race, and marital status.

$* p<.05$.

Randomization was assessed by comparing the sociodemographic characteristics of patients enrolled in both arms. Contingency table statistics and $t$ tests for means were used to compare interviewees from both arms. The data were analyzed with SPSS 12.0 (SPSS; Chicago, IL).

\section{Results}

Between September 2002 and October 2003, 1,089 persons sought treatment in CENTRA-RIO/SES. Seven hundred thirty-five individuals were eligible, willing, and randomized before entering treatment: ACASI $(n=367)$ and face-to-face interview $(n=368)$. There were 211 ineligible subjects. Among eligible cases, 125 refused to participate or left the facility before assessment. The most frequent reasons reported among those who refused to participate were: lack of time, no interest, and fear of being tested (although they were informed that blood test was not a requirement).

As shown in Table 1, the sociodemographic data for the two arms were quite balanced. Participants' ages ranged from 18 to 93 years old $(M=36, S D=10), 84.9 \%$ were male, $48.5 \%$ were White, $51.9 \%$ were Catholic, $52.6 \%$ had no spouse or stable partner, and $52.6 \%$ had less than seventhgrade education. These sociodemographic characteristics are similar to those of the overall population of patients on treatment at CENTRA-RIO/SES (Bastos, Simoes, Hacker, Malta, \& Metzger, 2004; Simoes et al., 2002).

Table 2 reports a comparison of responses to questions about the types of substances used during the prior 30 days. As can be seen, for each drug examined, the absolute rates of reporting were higher among those assigned to the ACASI mode of assessment. Adjusted and unadjusted ORs and confidence intervals were calculated to identify statistically significant differences in the rates of disclosure between the two study arms. For 7 of 10 substances, the difference was significantly higher. Adjusted ORs, controlling for age, gender, education, race, marital status, and income, revealed the same pattern of findings.
Sexual risk behaviors are reported in Table 3. Except for reports of having sexual intercourse (vaginal, anal, or oral sex, with a same-gender partner or not, in the past 6 months) and having multiple partners, reports of engaging in risk behaviors were higher among participants assigned to the ACASI arm. Lower rates were reported among those assigned to the ACASI arm for reporting being sexually active and having been tested for HIV.

\section{Discussion}

This article reports data from the first assessment of the performance characteristics of ACASI in Brazil and the largest examination of this method of data collection conducted outside the United States, among drug users. The data are confirmatory in that they provide evidence of impact similar to that reported in prior U.S. studies cited before: those assigned to the ACASI arm of the study reported more drug use and sexual risk behaviors.

Of particular importance are the significantly higher rates of commercial sex and MSM behavior reported among those assigned to ACASI assessment. The higher rates of reporting of these two high-risk behaviors via ACASI are consistent with those in prior studies (Des Jarlais et al., 1999; Macalino, Celentano, Latkin, Strathdee, \& Vlahov, 2002; Metzger et al., 2000). These behaviors appear to play an increasingly important role in the spread of HIV in Brazil and worldwide. A recent report by the Brazilian Ministry of Health (2004) shows an increasing incidence of AIDS cases due to heterosexual intercourse. Szwarcwald, Bastos, Barcellos, Esteves, and Castilho (2001) report on the geographic spread of heterosexual cases of AIDS among men and women in Rio de Janeiro, affecting women from disadvantaged communities. Among injecting drug users from Rio de Janeiro, Hacker et al. (2005) showed that male homosexual intercourse was a key risk factor for HIV infection among male injectors. 
Conversely, some behaviors had higher rates of endorsement in the interviewer mode. Specifically, interviewers were more likely to receive reports of sexual activity and previous testing for HIV. These responses highlight a central issue in understanding the utility of ACASI. Both sexual activity and prior HIV testing can be considered "socially desirable." In the current society's comprehension, being sexually inactive may be seen as an embarrassment and is not a well-accepted behavior. In the same way, in the specific context within which the interview occurred, a health care and drug treatment facility might create "expectations" felt by patients as "desirable," such as showing prior blood tests. Thus, "have been tested for HIV" and being "sexual active" fit a profile of "social desirability," and respondents might have felt more comfortable not reporting this "more acceptable behavior" in ACASI.

Despite the fact that all subjects sought drug treatment and were interviewed within a drug treatment facility, drug use was reported less often in the face-to-face method. Although the use of alcohol and marijuana was reported more often in the ACASI method, these were the only drugs for which no significant difference between methods was found. The reason for the similar disclosure of use of these two drugs by either method lies in their degree of social acceptability. Alcohol is a legal drug, and marijuana, although an illicit drug, is widely used.

In a study carried out in San Francisco with a sample of 2,881 men who have sex with men, crack use was reported at a significantly higher rate in telephone ACASI mode versus human interview telephone mode, whereas reports of marijuana use were similar in both methods (Gribble et al., 2000). These findings parallel our results wherein significantly higher rates of cocaine use were reported by those assessed via ACASI. It is likely that reports of the use of drugs with less social stigma will not be enhanced by the use of the ACASI method.

Because the study was conducted in a language and cultural setting different from that in prior studies of the ACASI method, the findings expand our understanding of the factors that influence disclosure and the interplay of global patterns of stigmatization vis-à-vis the unique characteristics of Brazilian culture. In this study, we wanted to test whether differing cultural sensitivities and definitions of acceptable behavior would result in a pattern of responses different from those seen in previous research conducted in different cultural settings. In fact, the data reported here point to remarkable similarities in the reporting of socially undesirable behaviors despite significant cultural differences. A companion article by our research group has recently addressed another key aspect of the implementation of ACASI in our setting_-good acceptability (Simoes et al., in press).

Some limitations of the study should be considered. Randomization strategy (1:1 on an alternative sequential basis) could have resulted in bias due to periodic changes in the characteristics of patients seeking treatment at the center. Notwithstanding, randomization resulted in two very com- parable groups. The differences in responses between the two assessment approaches cannot be attributed to sociodemographic factors. Analyses directed at identifying participant characteristics that could explain the findings resulted in negligible differences between the two arms. In addition, the characteristics of the participants in this study are consistent with those found in a previous evaluation of the population seeking treatment in this facility (Bastos et al., 2004; Simoes et al., 2002).

Although the sample size is large, the generalizability of the findings is limited because the study took place in a single drug treatment center. Despite the consistency of the findings with prior research, it is not possible to extrapolate our findings to those of other treatment settings. Finally, all of the data reported here are self-reported-this has historically raised concerns regarding validity (Des Jarlais et al., 1999; Macalino et al., 2002; Perlis et al., 2004). Again, the consistency of these data with those of prior studies suggests that the data can be considered informative. It is important to note that the literature has assumed that higher rates of reporting of socially undesirable behaviors have higher "face validity."

This article addresses the comparability of two interview methods using a single RAB in its Portuguese version. Future analyses would address more complex issues, such as the cross-cultural interpretation of ACASI and interview modes of RAB in the context of American and Brazilian drug treatment centers. Multicenter studies should be developed, with the aim to control for within-subject and betweensubject variability of responses, considering both the nature of drug treatment centers and the broad cultural context. Such issues are discussed at length by Giesinger (2000).

The findings of this study highlight the important role of ACASI in collecting data necessary for program planning and development. ACASI respondents reported a higher rate of drug use and a broader range of drug types. These findings clearly have an impact on the types of treatment services provided by the program. The role of ACASI assessment in the development of individual treatment plans is less clear. The confidential nature of assessment must be seen as the factor that leads to increased reporting. If individual responses to ACASI were used as the basis for an individual's treatment, the confidential nature of the assessment process may have been perceived as no different from that of an interview conducted by an interviewer. The use of the ACASI method in individualized treatment planning needs to be evaluated.

Despite the above-mentioned limitations, the use of ACASI in assessing risks can (and has) have a major role in program design and development. The impact of the present findings is important, considering the need to encourage comprehensive prevention programs for drug users in Brazil (Bastos et al., 2000; Oliveira et al., 1999) that fully integrate prevention and care (Bastos et al., 2000; Metzger \& Navaline, 2003a, 2003b). 


\section{Acknowledgments}

This research was supported by grant 914/BRA/3014 from the Brazilian Ministry of Health-HIV/STD National Coordination and the Health Department of Rio de Janeiro State Government, with additional funds from the HIV Prevention Research Division, University of Pennsylvania. We would like to acknowledge the following persons and institutions for their contributions to this research: Lucia Beck, Claudete Bueno, Rosana Silva, Carlos Silva, Rita Barcelos, Nadima Bernacchi, and Tereza Knett (ACORI); Rosa Ferreira and Eliane Campagnuci (Sao Francisco de Assisi Hospital); Eduardo Barbosa (Sao Sebastiao Institute; in memoriam); Mariana Hacker (Oswaldo Cruz Foundation); the CENTRA-RIO/SES team; and the people who took part in the research.

\section{References}

Bastos, F. I., Lowndes, C. M., Castello-Branco, L. R., Linhares-deCarvalho, M. I., Oelemann, W., Bernier, F., et al. (2000, June). Sexual behaviour and infection rates for HIV, blood-borne and sexually transmitted infections among patients attending drug treatment centers in Rio de Janeiro, Brazil. International Journal of STD \& AIDS, 11, $383-392$.

Bastos, F. I., Simoes, A. M., Hacker, M., Malta, M., \& Metzger, D. S. (2004). Drug use patterns among new admissions to a substance treatment center in Rio de Janeiro, Brazil (poster). Sixty-fifth annual scientific meeting, College on Problem of Drug Dependence, CPDD and 2004 NIDA International Forum, Puerto Rico, EUA.

Brazilian Ministry of Health. (2004). Epidemiological Week-01 a 52 January to December 2003. Epidemiological Bulletin-AIDS (Portuguese), XVIII, 26-37.

Davis, L. J., \& Morse, R. M. (1991). Self-administered alcoholism screening test: A comparison of conventional versus computeradministered formats. Alcoholism, Clinical and Experimental Research, $15,155-157$.

Des Jarlais, D. C., Paone, D., Milliken, J., Turner, C. F., Miller, H., Gribble, J., et al. (1999, May). Audio-computer interviewing to measure risk behaviour for HIV among injecting drug users: A quasi-randomised trial. Lancet, 353, 1657-1661.

Ellen, J. M., Gurvey, J. E., Pasch, L., Tschann, J., Nanda, J. P., \& Catania, J. (2002). A randomized comparison of A-CASI and phone interviews to assess STD/HIV-related risk behaviors. Journal of Adolescent Health, $31,26-30$.

Geisinger, K. F. (2000, Apr). Psychological testing at the end of the millennium: A brief historical review. Professional Psychology, Research and Practice, 31, 117-118.

Gribble, J. N., Miller, H. G., Cooley, P. C., Catania, J. A., Pollack, L., \& Turner, C. F. (2000). The impact of T-ACASI interviewing on reported drug use among men who have sex with men. Substance Use and Misuse, 35, 869-890.

Hacker, M. A., Friedman, S. R., Telles, P. R., Teixeira, S. L., Bongertz, V., Morgado, M. G., et al. (2005). The role of "long-term" and " new" injectors in a declining HIV/AIDS epidemic in Rio de Janeiro, Brazil. Substance Use and Misuse, 40, 1-31.

Jennings, T. E., Lucenko, B. A., Malow, R. M., \& Dévieux, J. G. (2002). Audio-CASI vs. interview method of administration of an HIV/STD risk of exposure screening instrument for teenagers. International Journal of STD \& AIDS, 13, 781-784.
Kissinger, P., Rice, J., Farley, T., Trim, S., Jewitt, K., Margavio, V., et al. (1999). Application of computer-assisted interviews to sexual behavior research. American Journal of Epidemiology, 149.

Locke, S. E., Kowaloff, H. B., Hoff, R. G., Safram, C., Popovsky, M. A., Cotton, D. J., et al. (1992, September 9). Computer-based interview for screening blood donors for risk of HIV transmission. Journal of the American Medical Association, 268, 1301-1305.

Lucas, R. W., Mullin, P. J., Luna, C. B. X., \& McInroy, D. C. (1977). Psychiatrists and a computer as interrogators of patients with alcohol-related illnesses: A comparison. British Journal of Psychiatry, 131, 160-167.

Macalino, G. E., Celentano, D. D., Latkin, C., Strathdee, S. A., \& Vlahov, D. (2002, Oct). Risk behaviors by audio computer-assisted selfinterviews among HIV-seropositive and HIV-seronegative injection drug users. AIDS Education and Prevention, 14, 367-378.

Mensch, B. S., Clark, W. H., \& Anh, D. N. (2003, Dec). Adolescents in Vietnam: Looking beyond reproductive health. Studies in Family Planning, 34, 249-262.

Metzger, D. S., Koblin, B., Turner, C., Navaline, H., Valenti, F., Holte, S., et al. (2000). Randomized controlled trial of audio computer-assisted self-interviewing: Utility and acceptability in longitudinal studies. American Journal of Epidemiology, 152, 99-106.

Metzger, D. S., \& Navaline, H. (2003a, Dec). HIV prevention among injection drug users: The need for an integrated models. Journal of Urban Health, 80 (4 Suppl 3), iii59-iii66 (Review).

Metzger, D. S., \& Navaline, H. (2003b). Human immunodeficiency virus prevention and the potential of drug abuse treatment. Clinical Infectious Diseases, 37 (Suppl 5), S451-S456.

Mills, A., Williams, M. L., Gordon, C., Young, P., Rusek, R., \& Signes, C. A. (1996). Reliability and validity of an Audio-CASI data collection instrument and evaluation of comparability with face-to-face interviewing. Final report to the National Institute on Drug Abuse (NIDA) community research branch.

Millstein, S. G. (1987). Acceptability and reliability of sensitive information collected via computer interview. Educational and Psychological Measurement, 47, 523-531.

Millstein, S. G., \& Irwin, C. E. (1983, Nov). Acceptability of computeracquired sexual histories in adolescent girls. Journal of Pediatrics, 103 , $815-819$.

Navaline, H. A., Snider, E. C., Petro, C. J., Tobin, D., Metzger, D. S., Alterman, A. I., et al. (1994). Preparations for AIDS vaccine trials. An automated version of the risk assessment battery (RAB): Enhancing the assessment of risk behaviors. AIDS Research and Human Retroviruses, 10 (Suppl 2), 281-283.

Newman, J. C., Des Jarlais, D. C., Turner, C. F., Gribble, J., Cooley, P., \& Paone, D. (2002, Feb). The differential effects of face-to-face and computer interview modes. American Journal of Public Health, 92, 294-297.

Oliveira, M. L., Bastos, F. I., Telles, P. R., Yoshida, C. F., Schatzmayr, H. G., Paetzold, U., et al. (1999, Sep). Prevalence and risk factors for $\mathrm{HBV}, \mathrm{HCV}$ and HDV infections among injecting drug users from Rio de Janeiro, Brazil. Brazilian Journal of Medical and Biological Research, 32, 1107-1114.

Perlis, T. E., Des Jarlais, D. C., Friedman, S. R., Arasteh, K., \& Turner, C. F. (2004, Jul). Audio-computerized self-interviewing versus face-to-face interviewing for research data collection at drug abuse treatment programs. Addiction, 99, 885-896.

Riley, E. D., Chaisson, R. E., Robnett, T. J., Vertefeuille, J., Strathdee, S. A., \& Vlahov, D. (2001). Use of audio computer-assisted self-interviews to assess tuberculosis-related risk behaviors. American Journal of Respiratory and Critical Care Medicine, 164, 82-85.

Sattah, M. V., Supawitkul, S., Dondero, T. J., Kilmarx, P. H., Yong, N. L., Mastro, T. D., et al. (2002). Prevalence of and risk factors for methamphetamine use in northern Thai youth: Results of an audiocomputer-assisted self-interviewing survey with urine testing. Addiction, 97, 801-808.

Simoes, A. M., \& Bastos, F. I. (2004, Sep-Oct). Audio ComputerAssisted Interview: A new technology in the assessment of sexually 
transmitted diseases, HIV, and drug use (Portuguese). Cadernos de Saude Publica, 20, 1169-1181.

Simoes, A. M., Beck, L. M., Lemos, C. J., Silva, C. B., Bueno, C. M., \& Metzger, D. S. (2002). HIV risk behavior among drug users entering in treatment in Rio De Janeiro, Brazil (poster). XIV International AIDS Conference, July 7-12, Barcelona, Spain.

Szwarcwald, C. L., Bastos, F. I., Barcellos, C., Esteves, M. A., \& Castilho, E. A. (2001). Spatial-temporal modeling: Dynamics of the AIDS epidemic in the municipality of Rio de Janeiro, Brazil, 1988-1996 (Portuguese). Cadernos de Saude Publica, 17, 1123-1140.

Turner, C. F., Ku, L., Rogers, S. M., Lindberg, L. D., Pleck, L. D., \& Sonenstein, F. L. (1998, May). Adolescent sexual behavior, drug use, and violence: Increased reporting with computer survey technology. Science, 280, 867-873.

Van de Wijgert, J., Padian, N., Shiboski, S., \& Turner, C. (2000). Is audio computer-assisted self-interviewing a feasible method of surveying in Zimbabwe? International Journal of Epidemiology, 29, $885-890$.

Van Griensven, F., Supawitkul, S., Kilmarx, P. H., Limpakarnjanarat, K., Young, N. L., Manopaiboon, C., et al. (2001, July). Rapid assessment of sexual behavior, drug use, human immunodeficiency virus, and sexually transmitted diseases in northern Thai youth using audiocomputer-assisted self-interviewing and noninvasive specimen collection. Pediatrics, 108, E13. 